\title{
Study of photon emission with the fission event generator FREYA
}

\author{
Ramona Vogt ${ }^{1,2, \star}$ and Jørgen Randrup ${ }^{3, \star \star}$ \\ ${ }^{1}$ Nuclear and Chemical Sciences Division, Lawrence Livermore National Laboratory, Livermore, CA 94551, \\ USA \\ ${ }^{2}$ Physics Department, University of California at Davis, Davis, CA 95616, USA \\ ${ }^{3}$ Nuclear Science Division, Lawrence Berkeley National Laboratory, Berkeley, CA 94720, USA
}

\begin{abstract}
The event-by-event fission model FREYA is employed to study photon observables. The model has been expanded beyond the simple statistical photon emission reported previously to include the discrete RIPL-3 lines. We update the earlier results and discuss the sensitivity of the results to the FREYA input parameters.
\end{abstract}

\section{Introduction}

The computational model FREYA [1-7] generates complete fission events, i.e. it provides the full kinematic information on the two product nuclei as well as all the emitted neutrons and photons. In its development, an emphasis had been put on speed so that large event samples can be generated fast. To make FREYA events as realistic as possible, FREYA relies on experimental data supplemented by simple physics-based modeling. In its standard version, to treat a given fission case, FREYA needs the fission fragment mass distribution $Y(A)$ and the total kinetic energy $\operatorname{TKE}(A)$ for the relevant energy. $Y(A)$ is taken either directly from the measured yields or as a five-Gaussian fit to the data which makes it possible to parameterize its energy dependence [3].

In order to generate an event, FREYA first selects the mass split based on $Y(A)$. The fragment charges are then sampled from the normal distributions suggested by experiment [3]. The linear and angular momenta of the two fragments and their internal excitations are subsequently sampled. After their formation, the fully accelerated fragments de-excite first by neutron evaporation and then by photon emission. In addition to spontaneous fission, FREYA treats neutron-induced fission up to $E_{n}=20 \mathrm{MeV}$; the possibility of pre-fission evaporation is considered as well as pre-equilibrium neutron emission (which plays a role at the highest energies). For full details, see Refs. [1-7].

In the new version of FREYA [7], we modulate the statistical emission of photons by the giant dipole resonance (GDR). Each photon emission reduces the total fragment angular momentum by $1 \hbar$. Statistical photons are emitted isotropically with an energy sampled from a black-body spectrum with the GDR form factor,

$$
\frac{d M_{\gamma}}{d E_{\gamma}} \sim \frac{\Gamma_{\mathrm{GDR}}^{2} E_{\gamma}^{2}}{\left(E_{\gamma}^{2}-E_{\mathrm{GDR}}^{2}\right)^{2}-\Gamma_{\mathrm{GDR}}^{2} E_{\gamma}^{2}} E_{\gamma}^{2} \mathrm{e}^{-E_{\gamma} / T}
$$

\footnotetext{
$\star$ e-mail: vogt2@1lnl.gov

${ }^{\star}$ e-mail: jrandrup@lbl.gov
} 
where $T$ is the temperature. The position of the resonance is taken as $E_{\mathrm{GDR}} / \mathrm{MeV}=31.2 / A^{1 / 3}+$ $20.6 / A^{1 / 6}$ [8], while its width is $\Gamma_{\mathrm{GDR}}=5 \mathrm{MeV}$. Note that in Ref. [4], photon emission assumed black-body radiation only with a spectrum of the form $d M_{\gamma} / d E_{\gamma} \sim E_{\gamma}^{2} \mathrm{e}^{-E_{\gamma} / T}$. The GDR has the effect of making the photon spectrum harder.

In addition, we now include the low energy photon lines from the RIPL-3 database [9]. The RIPL-3 library tabulates a large number of discrete electromagnetic transitions for nuclei throughout the nuclear chart but complete information is available for only relatively few of them. However, as we will discuss, by invoking certain assumptions it is possible to construct, for each product species, a table of the possible decays from the lowest discrete levels, i.e. the level energies $\left\{\varepsilon_{\ell}\right\}$, their half-lives $\left\{t_{\ell}\right\}$, and the branching ratios of their various decays. Then whenever the fragment de-excitation leads to an excitation energy below any of the tabulated levels, FREYA switches to a discrete cascade based on the RIPL-3 data. The discrete cascade is continued until the half-life $t_{\ell}$ exceeds the specified value of $t_{\max }$ or the ground state is reached. These lines have the most immediate impact on the low energy end of the photon spectrum, $E_{\gamma}<1 \mathrm{MeV}$.

The RIPL-3 data files are organized by element, with one file for each $Z$ value. Each such file contains similarly structured listings for those isotopes of that element for which data exist. For any tabulated nucleus, we seek to include all levels "in a complete level scheme", as indicated in the isotope header line on the data file. Each listed level $\ell$ may decay into a total of $n(\ell)$ lower levels $\left\{\ell^{\prime}\right\}$ and the associated relative transition rate $P\left(\ell \rightarrow \ell^{\prime}\right)$ is indicated for each one, if available. Often the rate for a listed transition is not given and the corresponding transition is then ignored.

However, if all the decay rates from a given level $\ell$ are missing we assign decay rates from that level to all of the lower levels $\ell^{\prime}$ based on a simple phase-space consideration:

$$
P\left(\ell \rightarrow \ell^{\prime}\right) \sim\left[E_{\ell}-E_{\ell^{\prime}}\right]^{2} \exp \left[-\left(J_{\ell}-J_{\ell^{\prime}}\right)^{2} / 2 d_{J}^{2}\right]
$$

Here $E_{\ell}$ is the energy of level $\ell, J_{\ell}$ is its listed spin, and we use $d_{J}=1$. It should be recognized that there are many more added transitions (201568) than tabulated transitions (75809). That is primarily because a level for which there are tabulated decay rates tends to decay to only some of the levels below it, whereas a level without tabulated decay rates is allowed to decay to any level below it. Furthermore, levels without tabulated decay rates tend to be high-lying and thus have many lower levels.

In the previous gamma decay procedure, the product nucleus first made statistical radiation (while conserving the angular momentum) until the yrast line was reached and then proceeded towards the ground state by collective emission. In order to incorporate the discrete decays, we take note of the highest tabulated discrete energy $E_{\ell}$ and then follow the earlier procedure until the total excitation has fallen below that value. (It should be noted that the statistical decay procedure was modified so that the angular momentum is reduced by one unit for each emission in order to better emulate the predominantly $E 1$ and $M 1$ character of those transitions.) In order to ensure that the nucleus arrives at a tabulated level, the energy of the last statistical transition is increased (slightly) so that the final state is the closest lower-lying level. Further de-excitation is then carried out by means of the discrete rates, as described above.

If there are no RIPL-3 transitions available for a given fragment, the de-excitation occurs by emission of "collective" photons along the yrast line. Each emitted photon reduces the angular momentum by $2 \hbar$, as in Ref. [4].

In either case, photon emission continues until the energy is below some specified minimum energy, $g_{\min }$, which is generally detector dependent.

FREYA contains a number of adjustable parameters that control various physics aspects: 
Theory-4

$d \mathrm{TKE}$, an overall shift of $\overline{\operatorname{TKE}}(A)$ relative to the input $\operatorname{TKE}(A)$, used to adjust to the average neutron multiplicity $\bar{v}$;

$e_{0}$, the overall scale of the Fermi-gas level density parameters;

$x$, the advantage in excitation energy given to the light fragment;

$c_{T}$, the relative statistical fluctuation in the fragment excitations;

$c_{S}$, the ratio of the "spin temperature" to the "scission temperature";

$g_{\min }$, the minimum energy photon registered and counted by FREYA;

$t_{\max }$, the maximum half-life of a level during the photon decay process which stops when the level half-life exceeds $t_{\max }$.

The three parameters that most affect photon observables are $c_{S}, g_{\min }$ and $t_{\max }$. We give some examples here of how changing these parameters affects the photon results. The value of $c_{S}$ governs the amount of rotational energy in the system and thus strongly affects most observables. The values of $g_{\min }$ and $t_{\max }$ are detector dependent, related to the energy threshold for photon detection and the time gate for real detectors so that the results for a particular experimental configuration depend on this information to make a comparison. We will show the effect of changing $c_{S}$ and $g_{\min }$ on the total photon energy and multiplicity as a function of fragment mass. However, the effect of changing $t_{\max }$ on the photon observables is more subtle. Therefore, we will show the changes in the photon multiplicity relative to a maximum time of $\sim 5 \mu \mathrm{s}$ for several different values of $g_{\min }$ and $c_{S}$.

\section{Parameter sensitivity}

We first discuss the effect on photon observables caused by changing the parameter $c_{S}$, effectively changing the rotational energy in the system. The other parameters are kept fixed while $c_{S}$ is varied. We choose to show results for $c_{S}=0.2,0.8,1.4$ and 2.0. The value of 0.8 is rather close to the best fit value of 0.87 for ${ }^{252} \mathrm{Cf}(\mathrm{sf}$ ) [10] but here we take equal spacings between values instead of using a particular fitted value. The value 0.2 is taken as a lower bound, reducing the rotational energy in the system without removing it altogether. The two values larger than unity serve to illustrate the range of the effect.

Figure 1 presents the total photon energy and photon multiplicity as functions of fragment mass for the four values of $c_{S}$. The statistical uncertainties on the one million FREYA events used to obtain the results for each value of $c_{S}$ are shown. They are typically very small. For example, the uncertainties on the energy in Fig. 1a) are typically negligible, only appearing far into the tails of the fragment distributions, $A<90$ and $A>160$, where only a few hundred events are sampled. Because the dip in the mass yield at symmetry is reduced for ${ }^{252} \mathrm{Cf}(\mathrm{sf})$ relative to other asymmetrically fissioning isotopes, the uncertainties near $A=126$ are reduced relative to those in the tails.

In Fig. 1a), it can be seen that the photon energy simply grows by approximately $0.5 \mathrm{MeV}$ when $c_{S}$ increases by 0.6. There is no visible mass dependence on changing $c_{S}$. However, the photon multiplicity does show some modification of the fragment mass dependence with increasing $c_{S}$, see Fig. 1b). While the general trend is the same for all values of $c_{S}$, there is a larger increase in the multiplicity for the heavy fragment while the dip at the $A \sim 132$ doubly-closed shell deepens with increasing $c_{S}$. In addition, there appears to be a rather flat plateau for the light fragment masses $100<A<120$ for $c_{S}=0.2$ that acquires a positive slope as $c_{S}$ increases. 

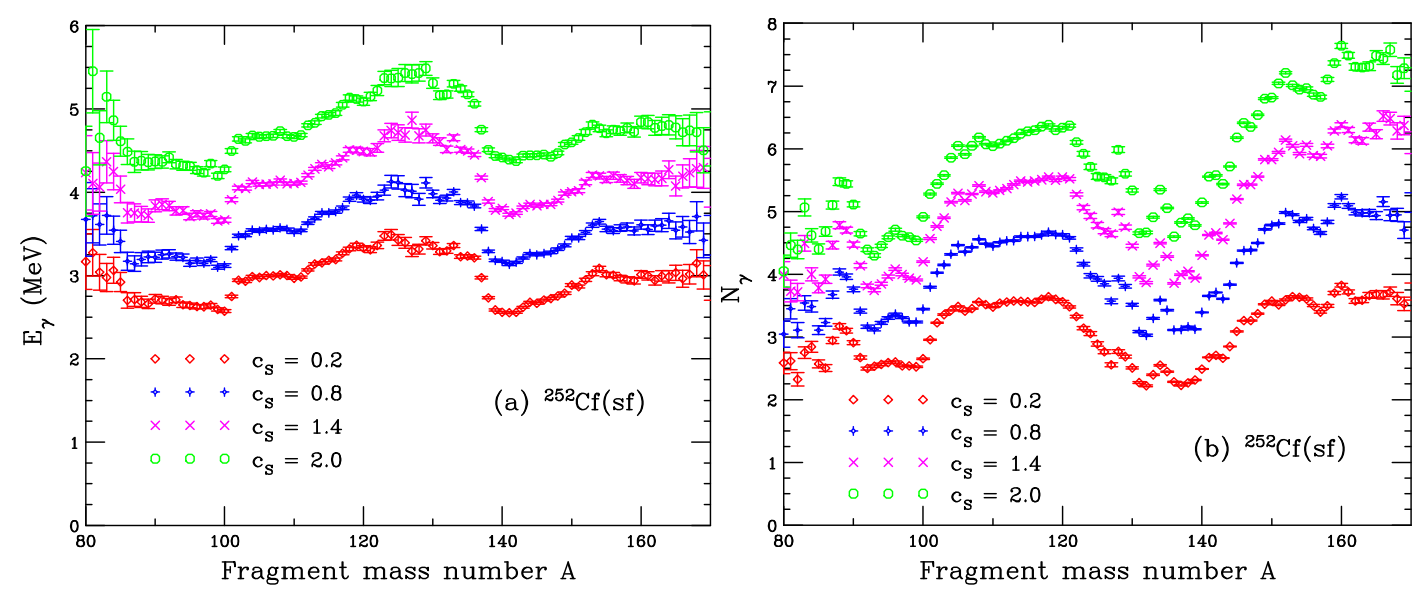

Figure 1. The total photon energy (a) and photon multiplicity (b) as functions of fragment mass for ${ }^{252} \mathrm{Cf}(\mathrm{sf})$ from FREYA calculated for four different values of the parameter $c_{S}$ which sets the amount of the fragment spin. Results are shown for $c_{S}=0.2$ (red), 0.8 (blue), 1.4 (magenta) and 2.0 (green). Each calculation is based on one million FREYA events. The statistical uncertainties in the calculations are shown.

We note, however, that these results are calculated assuming that no other parameter value changes. Thus, if the total excitation energy is held fixed, increasing the rotational energy, as happens for increased $c_{S}$, then less energy is available for neutron emission. Thus increasing $c_{S}$ while keeping the other parameters fixed will decrease the average neutron multiplicity. Although this could be compensated for by changing the value of $d \mathrm{TKE}$, one has to be careful to adjust it within reasonable physics limits to not spoil the agreement of the calculation with other measured observables.
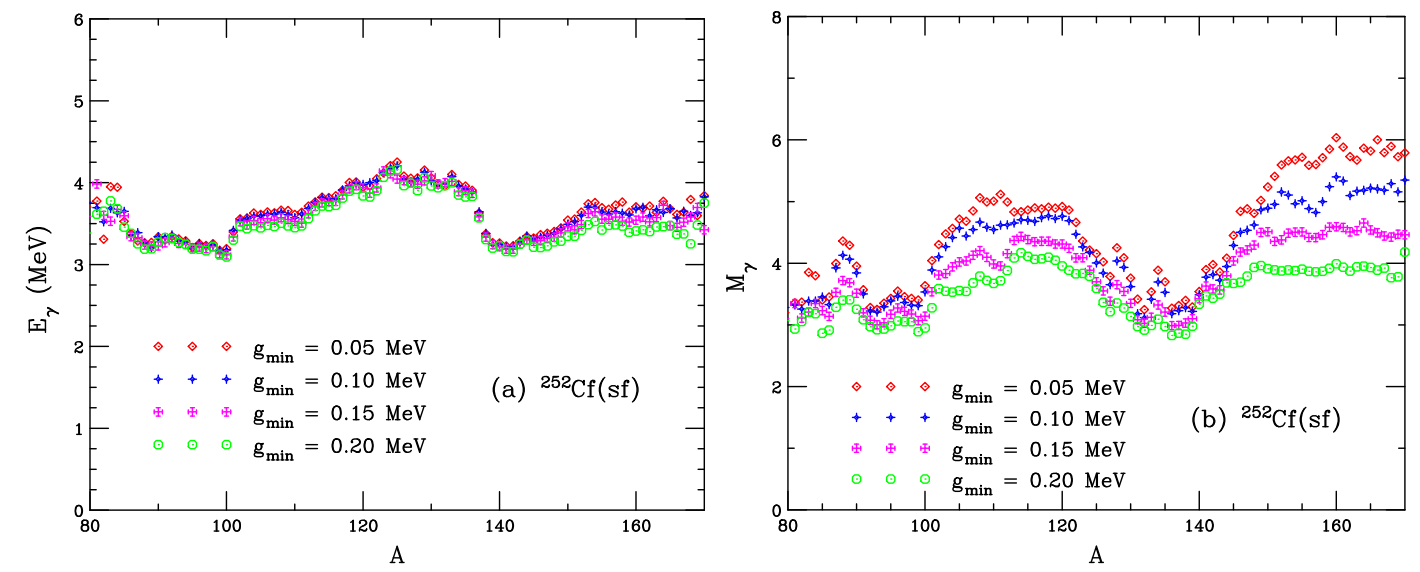

Figure 2. The total photon energy (a) and photon multiplicity (b) as functions of fragment mass for ${ }^{252} \mathrm{Cf}(\mathrm{sf})$ from FREYA calculated for four different values of the parameter $g_{\min }$, the minimum photon energy detected. Results are shown for $g_{\min }=0.05$ (red), 0.10 (blue), 0.15 (magenta) and 0.20 (green). Each calculation is based on one million FREYA events. 
Figure 2 shows the result of changing $g_{\min }$ between 0.05 and $0.2 \mathrm{MeV}$. Since the results overlap significantly here, we do not show the statistical uncertainties. However, we note that they would be similar to those shown in Fig. 1. The change in total photon energy, shown in Fig. 2a), is negligible since the largest value, $g_{\min }=0.2 \mathrm{MeV}$, makes, on average, a $\sim 5 \%$ change in the photon energy emitted from a given fragment. The effect on the photon multiplicity is significant albeit not as large as changing $c_{S}$ by a factor of 10, between 0.2 and 2, as shown in Fig. 1b). As $g_{\min } \rightarrow 0$, more and more soft photons could be emitted, increasing the total multiplicity.

In Fig. 2b), we can see that the largest change in $M_{\gamma}$ with $A$ occurs for $A<100$ and $125<A<140$. Both are in the vicinity of closed nuclear shells where one expects reduced emissions due to the lower total excitation energy imparted to these fragments. The photon multiplicity from the heavy fragment appears to be the most affected by changing $g_{\min }$ with a $\sim 50 \%$ change in $M_{\gamma}$ at $A \sim 160$ relative to a $\sim 20 \%$ change near $A \sim 110$. The difference arises from the higher spin of the heavy fragment for the best fit value of $c_{S}$, see Ref. [7]. The higher spin implies larger rotational energy, a great probability of photon emission and thus higher photon multiplicity from the heavy fragments. Because the multiplicity dependence on $A$ is reduced as $g_{\min }$ increases, $M_{\gamma}$ may be expected to become approximately independent of $A$ at higher values of $g_{\min }$ where only statistical photon emission, generally higher energy, is possible. For the effect of increasing $g_{\min }$ up to $2 \mathrm{MeV}$ on the average values of $E_{\gamma}$ and $M_{\gamma}$, see Ref. [7].

With the addition of the RIPL-3 library discrete photon lines, the time window in which the detector operates can affect the measured photon spectrum, particularly at low energies. For example, if the photon cascade from a fission fragment lands on a long-lived isomeric state, the decay will not proceed further during the measurement time and no more photons will be emitted during the process. FREYA can populate such isomeric states based on the initial fragment distributions and neutron emission.

To show how these ratios could change with inputs, we look first at the cumulative photon multiplicity and total photon energy as a function of $t_{\max }$ for different values of $g_{\min }$ in Fig. 3a). We choose relatively low values of $g_{\mathrm{min}}$, from 0.05 to $0.20 \mathrm{MeV}$. Given the rather small differences between
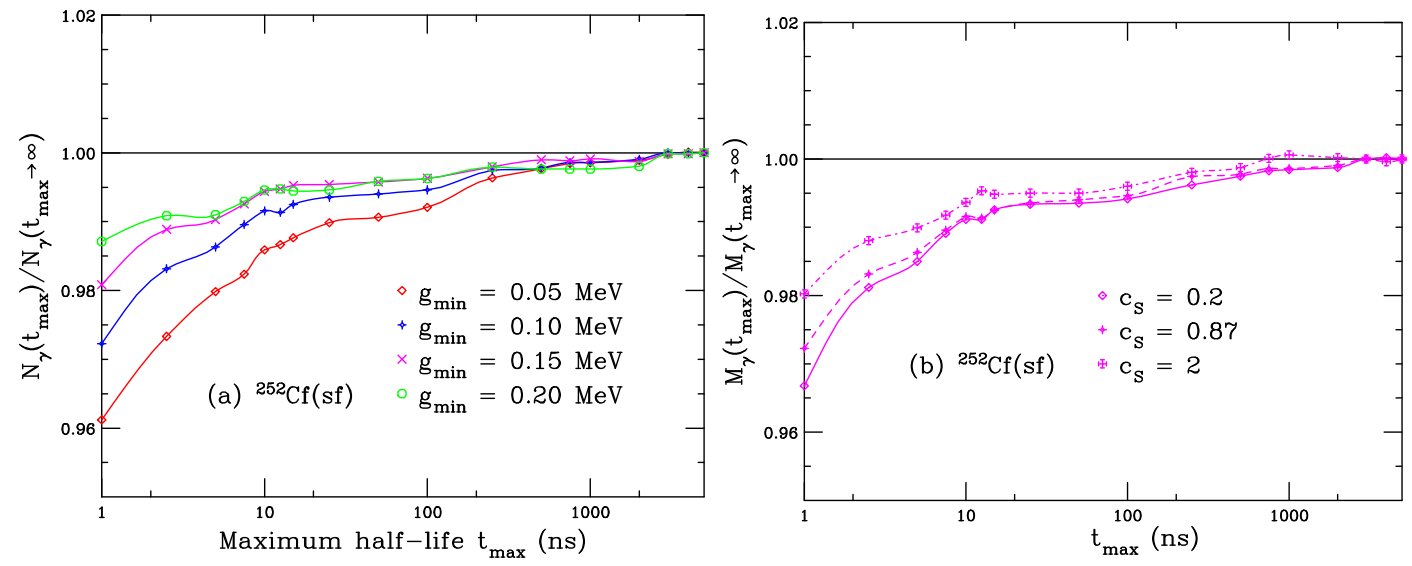

Figure 3. The total photon multiplicity relative to the maximum value at $t_{\max } \rightarrow \infty$ (effectively $t_{\max }=5 \mu \mathrm{s}$ ) for ${ }^{252} \mathrm{Cf}$ (sf) as a function of photon half-life $t_{\max }$ for (a) changing $g_{\min }$ ( $g_{\min }=0.05$ (solid), 0.10 (dashed), 0.15 (dot-dashed) and 0.20 (dotted) $\mathrm{MeV}$ ) and (b) changing $c_{S}\left(c_{S}=0.2\right.$ (solid), 0.87 , the default value (dashed) and 2.0 (dot-dashed)). Each point is from a calculation of one million FREYA events. 
$g_{\min }=0.15$ and $0.20 \mathrm{MeV}$, the results for higher values of $g_{\min }$ would become indistinguishable, especially for higher $t_{\max }$. The largest effect is on the multiplicity, shown in Fig. 3a), and for $t_{\max } \leq 100 \mathrm{~ns}$. The greatest difference is between $g_{\text {min }}=0.05 \mathrm{MeV}$ and $0.10 \mathrm{MeV}$. Higher values of $g_{\text {min }}$ have a reduced effect because the discrete levels tend to emit rather soft photons. The effect on the cumulative total photon energy is minimal.

Figure $3 \mathrm{~b}$ ) shows the dependence on the cumulative photon multiplicity on $t_{\max }$ for several values of $c_{S}$. The middle ratio is the best fit value from the ${ }^{252} \mathrm{Cf}(\mathrm{sf})$ fit [10], $c_{S}=0.87$ and is the same as the results shown in Fig. 3a) for $g_{\min }=0.10 \mathrm{MeV}$. The other two values, $c_{S}=0.2$ and 2 are the upper and lower limits used in the calculations shown in Fig. 1. The dependence on $c_{S}$ is weaker than that on $g_{\min }$.

We do not show uncertainties here, the uncertainty on the average photon multiplicity over one million FREYA events is on the sub-percent level. However, some of the non-smooth behavior of the ratios may be due to such sub-percent fluctuations in the total multiplicity between FREYA runs.

The results presented here are only a relatively small subset of the observables we have studied. To see all the results as well as comparison to available data, see Ref. [7].

The work of R.V. was performed under the auspices of the U.S. Department of Energy by Lawrence Livermore National Laboratory under Contract DE-AC52-07NA27344. The work of J.R. was performed under the auspices of the U.S. Department of Energy by Lawrence Berkeley National Laboratory under Contract DE-AC0205CH11231. The authors also thank the United States Department of Energy National Nuclear Security Administration Office of Defense Nuclear Nonproliferation Research and Development for support for this work.

\section{References}

[1] J. Randrup and R. Vogt, Phys. Rev. C 80, 024601 (2009)

[2] R. Vogt and J. Randrup, Phys. Rev. C 84, 044621 (2011)

[3] R. Vogt, J. Randrup, D.A. Brown, M.A. Descalle, and W.E. Ormand, Phys. Rev. C 85, 024608 (2012)

[4] R. Vogt and J. Randrup, Phys. Rev. C 87, 044602 (2013)

[5] R. Vogt and J. Randrup, Phys. Rev. C 90, 064623 (2014)

[6] J. Randrup and R. Vogt, Phys. Rev. C 89, 044601 (2014)

[7] J. Randrup and R. Vogt, submitted to Phys. Rev. C [arXiv:1708.07312]

[8] B.L. Berman and S.C. Fultz, Rev. Mod. Phys. 47, 713 (1975)

[9] R. Capote et al., Nucl. Data Sheets 110, 3107 (2009)

[10] R. Vogt, A. Nicholson, J. Randrup, I. Gauld and S. Croft, Proc. $1^{\text {st }}$ ANS Advances in Nuc. Nonpro. Tech. and Policy, Santa Fe, NM, 2016, LLNL-CONF-690741 